\title{
Soziale Klassen und Klassenkonflikt: Zur Entwicklung und Wirkung eines Theoriestücks
}

\section{Ein persönlicher Bericht}

\section{Ralf Dahrendorf}

Universität Konstanz, Sozialwissenschaftliche Fakultät, Universitätsstr. 10, 7750 Konstanz 1

Zu s a m me n f a ss ung: In diesem Beitrag werden die Entstehung des Buches „Soziale Klassen und Klassenkonflikt“ und die Weisen seiner Rezeption im deutschen, angelsächsischen und italienisch-iberisch-lateinamerikanischen Kulturbereich aus der Sicht des Autors beschrieben und analysiert.

In der Zeit unmittelbar nach dem Krieg gab es die Soziologie als Studienfach nur an wenigen Orten. Auch wenn es sie an der Universität Hamburg gegeben hätte, wäre der Student aus einer universitätsfernen Familie ihr wohl nicht begegnet. Mein Studium begann mit Germanistik und Philosophie, konzentrierte sich dann stärker auf Philosophie, bis ein kluger akademischer Lehrer riet, der Sache eine solide Basis zu geben. Physik und Mathematik oder Klassische Philologie? Die letztere lag näher, wurde fast zum Hauptfach, bis mein Doktorvater in der Philosophie fragte, was mich denn im Leben sonst interessiert. Ich erzählte vom politischen Elternhaus, von der eigenen Tätigkeit im Sozialistischen Studentenbund. Der verständnisvolle Professor lenkte mich von der hellenistischen (Sozial-) Philosophie, über die ich arbeiten wollte, zum Autor einer Dissertation über hellenistische (Natur-)Philosophie, Karl Marx. Mit 22 Jahren promovierte ich 1952 über den „Begriff des Gerechten im Denken von Karl Marx".

Das Buch, das im folgenden Jahr auf Grund der Dissertation erschien (Marx in Perspektive), endet mit zwölf Thesen zur Marx-Kritik. Die Thesen 11 und 12 wurden damals ahnungslos, oder vielmehr ahnungsvoll, aber kenntnislos geschrieben:

„11. Es gibt nur eine Instanz zur Widerlegung von Marx’ sozialwissenschaftlichen Hypothesen und Vorhersagen: empirische Tatbestände, deren Urteil diese Hypothesen wie alle Annahmen der Sozialwissenschaften unterliegen.

12. Die wesentlichen und verfolgenswerten Beiträge des sozialwissenschaftlichen Teils des Marxschen Werkes sind

(a) Marx' Begriff der Klasse und die aus ihm hervorgehenden Annahmen;

(b) die Hypothese der Deriviertheit der Ideen; und vor allem

(c) die Hypothese der Logik sozialer Veränderungen.“

Auch der Entschluß, anschließend an die London School of Economics zu gehen, war noch ahnungs- voll, aber kenntnislos. Die Londoner Jahre führten in zweierlei Hinsicht zur Vertiefung der Absichten der Dissertation. Einmal fügten sie der akademischen manche reale Erfahrung hinzu. Das geschah durch die Dissertation über Unskilled Labour in British Industry und die damit verbundenen Beobachtungen in der Industrie. Es geschah wohl auch, weniger direkt aber nicht weniger wirksam, durch das Einatmen des spezifisch britischen Sinns für das Austragen sozialer Konflikte. Adversary politics ist ja ein sehr britisches Phänomen; schon die Übersetzung „Freund-Feind-Politik“ zeigt die ganz anderen deutschen Bewertungen. Konflikte anerkennen, regeln und austragen, und zwar Konflikte zwischen jeweils zwei Protagonisten, ist bis heute ein Grundzug der politischen Kultur des Landes.

Zum anderen brachten die Londoner Jahre wichtige akademische Begegnungen. Die mit Karl Popper gab der im strengen Sinn sozialwissenschaftlichen Absicht der Thesen zur Marx-Kritik Konturen und eine Sprache. Die Offene Gesellschaft und ihre Feinde, aber nicht minder die Logik der Forschung wurden zu intellektuellen Schlüsselerlebnissen. Die Begegnung mit Parsons - dem Parsons, der gerade The Social System veröffentlicht hatte führte im Gegenteil zu einer höchst kritischen Beziehung. Sie verstärkte die Absicht, Konflikt und nicht Konsensus, Wandel und nicht Integration ins Zentrum der Aufmerksamkeit zu rücken. Das kritische Sich-Reiben an Parsons wurde dabei zum befruchtenden Element (bis hin zu dem Aufsatz „Out of Utopia“, einem Resultat des Jahres 1957-58, in dem Parsons und ich beide am Center for Advanced Study in Palo Alto waren). An der London School of Economics veranstalteten wir Graduierten ein eigenes Seminar, in dem wir uns intensiv mit der "Interessengruppen-Theorie des Konflikts" befaßten.

So nahm ich im Juli 1954 gerne die angebotene Assistentenstelle am Frankfurter Institut für So- 
zialforschung an. Die Freude dauerte indes nicht lange. Desillusioniert kündigte ich nach vier Wochen. Der letzte Nagel im Sarg dieses Traums war ein Gespräch mit Horkheimer und Adorno über Habilitationsabsichten. Beide erklärten mir nicht nur, daß dafür noch viel, viel Zeit sei, sondern auch, daß es doch nicht nötig sei, die Leute (in Adenauers Bundesrepublik der 50er Jahre) mit so etwas wie „sozialen Klassen“ zu schockieren.

Zweieinhalb Jahre dauerte es dann in der Tat noch bis zur Habilitation, aber das Interesse am Thema erlahmte nicht. Dazu trug der Kontakt mit anderen Soziologen der Nachkriegsgeneration im „Industriesoziologen-Kreis“" (der sich kurioserweise häufig im Frankfurter Institut traf) bei, also mit Popitz, Bahrdt, Jüres und Kesting, mit Pirker und Lutz, mit Friedeburg und Teschner und Brandt. Helmut Schelsky, der sich vieler junger Soziologen fürsorglich annahm, veröffentlichte in diesen Jahren seine Analysen der ,nivellierten Mittelstands-

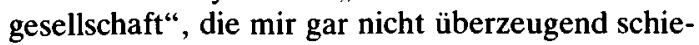
nen. Sein Hamburger Kollege Siegfried Landshut trieb die These noch weiter und behauptete, die klassenlose Gesellschaft sei zumindest im Westen bereits da. Zugleich fanden die vom Internationalen Soziologenverband angeregten Schichtungsuntersuchungen weite Verbreitung. Am Ende setzte ich mich hin und schrieb in wenigen Wochen das Buch Soziale Klassen und Klassenkonflikt in der industriellen Gesellschaft, das 1957 an der Universität des Saarlandes, an der ich nach dem Frankfurter Zwischenspiel eine Assistentenstelle gefunden hatte, als Habilitationsschrift angenommen wurde. Das Buch war allzu rasch geschrieben. Erst in der Muße des Center in Palo Alto gewann es wirklich Gestalt, und zwar auf englisch. Erst Class and Class Conflict in Industrial Society aus dem Jahre 1959 ist also das Theoriestück, von dem hier die Rede ist. Worum es dabei geht, läßt sich in wenigen einfachen Sätzen sagen. Es ist eine vornehme Aufgabe der Sozialwissenschaft, gesamtgesellschaftliche Entwicklungen zu verstehen. Der eindringlichste Versuch, dies zu leisten, ist der von Marx. Das gilt insbesondere für seine Theorie, wonach gesellschaftliche Veränderungen das Ergebnis von Auseinandersetzungen zwischen Gruppen (,Klassenkämpfen“) sind, die ihre Kraft aus den obwaltenden sozialökonomischen Umständen beziehen. Immer kämpft eine Klasse im Namen zukünftiger Möglichkeiten gegen eine andere, die vorhandene Strukturen verteidigt.

Allerdings sind die Prognosen, die aus der Marxschen Klassenkampftheorie folgen, in den entwik- kelten Industriegesellschaften nicht eingetroffen. Es hat keine immer zunehmende Polarisierung in zwei Klassen gegeben. Das politisch-sozialen Konflikten zugrundeliegende Sozialverhältnis ist nicht ausschließlich oder selbst vorwiegend das der Verfügung über industrielle Produktionsmittel geblieben. Die vorhergesagte („proletarische“) Revolution hat nicht stattgefunden, jedenfalls nicht dort, wo sie nach Marx hätte stattfinden müssen. Irgendetwas muß also falsch sein an der Marxschen Theorie.

Hier ist dann allerdings eine Entscheidung zu treffen. Man kann sich dazu entschließen, die Marxsche Theorie einfach zu vergessen. Das hat in gewisser Weise Schelsky getan. Es ist der unausgesprochene Hintergrund der Verflachung der Klassenanalyse zur Beschreibung der sozialen Schichtung. Man kann die Marxsche Theorie auch (historizistisch) gleichsam ablegen, zum historischen Phänomen mit gewissen zeitgenössisch relevanten Elementen erklären, und andere Analysen an ihre Seite stellen. Das ist die Grundtendenz der sogenannten Frankfurter Schule. Man kann schlieBlich daran gehen, die Marxsche Theorie in einem strengen Sinn zu überwinden, also sie durch eine bessere Theorie zu ersetzen. Das haben bedeutende Sozialwissenschaftler wie Theodor Geiger (Klassengesellschaft im Schmelztiegel) und Karl Renner (Wandlungen der modernen Gesellschaft) schon in den 40er Jahren getan. Darin lag auch meine Absicht.

Bei diesem Prozeß der Überwindung waren mir zwei Schritte besonders wichtig. Der erste ist methodisch. Viele Marxsche Irrtümer beruhen auf seinem philosophischen Dogmatismus. In hegelischer Sicht muß Wandel explosiv, revolutionär sein (während tatsächlich Revolutionen seltene Ausnahmeformen des Wandels sind). In hegelischer Sicht muß es eine systematische Zuspitzung von Konflikten geben (während der Prozeß sozialer Konflikte tatsächlich höchst variablen empirischen Bedingungen unterliegt und übrigens die „äußerste Not" weniger die ,äußerste Notwendigkeit der Revolution" als Lethargie signalisiert). In hegelischer Sicht muß irgendwann ein vollkommener Endzustand, eine "klassenlose Gesellschaft" erreicht werden (während vieles dafür spricht, daß im Horizont der Ungewißheit menschliche Geschichte mit ihren Möglichkeiten und Wechselfällen immer offen bleibt). Es gilt also, Sozialwissenschaft und Geschichtsphilosophie bei Marx zu trennen, d. h. von jedem Marxismus Abschied nehmen. 
Der zweite Schritt ist dann sozialwissenschaftlich. Spricht man nur mehr vom Marx, dem Klassiker der Soziologie, dann fällt vor allem seine Bindung an das eine Thema der Zeit, die (industrielle) Produktion, und in diesem Zusammenhang an das Rechtsverhältnis des Eigentums auf. „Produktivkräfte“ und „Produktionsverhältnisse" sind ebenso wie „Eigentum an Produktionsmitteln" und „Proletarier“" (d. h., im römischen Zensus, diejenigen, die nichts zu versteuern, sondern nur - zahlreiche Kinder haben) verräterische Begriffe. Um wirkliche Entwicklungen zu erklären, muß man die Theorie aus ihrer Zeitbindung befreien. Nicht Eigentum, sondern Verfügungsgewalt, Herrschaft ist wichtig; nicht nur die Produktion, sondern alle sozialen Zusammenhänge zeigen Herrschaftsstrukturen.

Von hier nimmt dann die Theorie der Konflikte ihren Ausgang. Sie befaßt sich mit der Entstehung von Herrschaftskonflikten, mit den Bedingungen ihres Verlaufs, mit ihrer Auswirkung auf soziale Wandlungen. Sie schließt eine Theorie der politischen Organisation ebenso ein wie eine Theorie der Revolution.

Das alles läßt sich in Class and Class Conflict nachlesen. Das Buch hat auch in seiner englischen Form noch viele Schwächen. Einige davon habe ich später zu reparieren versucht (z. B. in dem Vortrag „Conflict After Class“ aus dem Jahr 1967). Andere, die sich auf die soziale Basis von Konflikten beziehen, also die Begriffe der „Produktivkräfte“ und der „Produktionsverhältnisse“ zu verallgemeinern suchen, bleiben einstweilen in unveröffentlichten Manuskripten begraben. Aber hier ist ja weniger die Sache selbst, also die Theorie des sozialen Wandels durch Konflikt, als die Entstehung und Wirkung eines Buches das Thema, auch wenn die beiden zusammenhängen.

In Deutschland ist die Wirkung des Klassenbuches gering geblieben. Die erste Auflage von $2000 \mathrm{Ex}-$ emplaren ist zwar vergriffen, aber eine zweite Auflage, eine Übersetzung der endgültigeren englischen Version, ist nie erschienen. Auch hat das Buch nicht etwa eine nachhaltige Diskussion ausgelöst, wie das etwa für den Essay Homo Sociologicus gilt. Das hat wahrscheinlich etwas zu tun mit der deutschen Harmoniesehnsucht, die ja nicht etwa nur die politische Rechte (,nivellierte Mittelstandsgesellschaft"), sondern auch die politische Linke (,herrschaftsfreie Kommunikation“) kennzeichnet. Auch das durchaus verquere, nämlich entweder neurotische oder enthusiastische Verhältnis zu Marx mag hier eine Rolle spielen.
Einmal, auf dem Soziologentag von 1968, hat es eine Diskussion des Themas gegeben. Das war ein schwieriger Soziologentag zu einer schwierigen Zeit. (Ich war damals der Vorsitzende der Deutschen Gesellschaft für Soziologie und zu engagiert, um angesichts der unterschwelligen Emotionen der Diskussionen die nötige Distanz zu zeigen; das hat eher schon der Diskussionsleiter Heinrich Popitz mit seiner unnachahmlichen Ironie getan.) Unter dem Titel „Herrschaft, Klassenverhältnis und Schichtung" vertraten damals J. Bergmann, G. Brandt, K. Körber, E. Th. Mohl und C. Offe die These:

„Die unter dem Gesichtspunkt des sozialen Wandels dominante Form der Ungleichheit ist weniger in der vertikalen Dimension der Ungleichheit von Schichten und Klassen zu suchen als in der horizontalen Form der Disparität von Lebensbereichen, d. h. der ungleichgewichtigen Befriedigung der verschiedenen Lebensbedürfnisse."

Vor allem Claus Offe hat diesen Einwand (etwa in seinen Strukturproblemen des kapitalistischen Staates) auch sonst entwickelt. Er besagt, in frankfurterisch verstellter Sprache, nichts anderes als die simple These von Galbraith, daß es seinerzeit einen Widerspruch gab zwischen privatem Wohlstand und öffentlicher Armut. Viele Menschen hatten zwar einigermaßen hohe Einkommen, aber ihre Bildungschancen blieben begrenzt, ihre Umwelt blieb verpestet. Das alles, so Offe und andere, ruft Zweifel wach an der ,theoretischen Reichweite eines analytischen Modells, das nach wie vor mit der vorrangigen Relevanz von Schichtungs- und Klassenstrukturen für eine Theorie der gesamtgesellschaftlichen Entwicklung rechnet". Es ist wohl diese Art der Diskussion, die einen der Herausgeber der „Zeitschrift für Soziologie“ veranlaßt, von der Klassentheorie als einem ,relativ abgeschlossenen Theorie- und Analysestück“ zu sprechen.

Indes ist in den angelsächsischen Ländern die Wirkung des Klassenbuches nachhaltiger gewesen. Sie dauert bis auf den heutigen Tag. Das liegt nicht nur daran, daß vor allem Amerikaner etwas leichter „Klassiker“ ernennen als Europäer. In diesem Fall ist der „Klassiker Dahrendorf“ sogar nach Europa zurückgekehrt, wo Raymond Aron der französischen Ausgabe des Buches 1972 eine Einleitung voranstellte. „Dieses Werk, obgleich oder besser: weil - es ein Jugendwerk ist, das den Klassenbegriff bei Marx und seitherige Wandlungen der Sozialstruktur im Lichte jüngerer Theorien untersucht, kann auf Grund der Strenge der Analyse, der Kenntnis der Literatur, als klassisch be- 
zeichnet werden." In Ann Arbor, an der University of Michigan, ist es mir geschehen, daß ein Student mich fragte, ob mein Vater auch Soziologe gewesen sei, denn er habe ein 1959 erschienenes Buch über Klassenkonflikt von einem gewissen Dahrendorf zum Examensthema gehabt.

Die angelsächsische Wirkungsgeschichte des Klassenbuches geht weiter. Noch heute werden in jedem Jahr mehr englische Exemplare des Buches gekauft als je deutsche gedruckt worden sind. Dutzende von Dissertationen widmen sich dem Thema. Für dieses bleibende Interesse gibt es wahrscheinlich mindestens drei Gründe. Der erste liegt in einem methodischen Ansatz, der das Interesse an gesamtgesellschaftlichen Entwicklungen mit der Absicht der Erfahrungswissenschaft verbindet. Das ist ja nicht selbstverständlich. In Deutschland etwa gibt es, von den wichtigen Beiträgen Wolfgang Zapfs, Peter Floras und anderer zur Sozialberichterstattung abgesehen, vornehmlich erfahrungswissenschaftliche Mikrosoziologie und sozialphilosophische Makrosoziologie. Das fehlende dritte wird wohl auch nicht vermißt. Das ist in der angelsächsischen Denkkultur anders.

Der zweite Grund für das nachhaltige Interesse am Klassenbuch in England wie in den USA liegt in der außerordentlichen Dominanz von Talcott Parsons im Bereich dessen, was man so Theorie nennt. Da haben manche ein Korrektiv, vielleicht auch eine Alternative gesucht. Während es nun ganz abwegig wäre, für das Klassenbuch mehr in Anspruch zu nehmen, als daß es eine mögliche andere Richtung der Analyse andeutet, während das Buch also mit dem weitverzweigten systematischen Ansatz von Parsons nicht vergleichbar ist, galt und gilt es doch als Hinweis auf die Möglichkeit einer anderen Denkweise. Das war ja auch beabsichtigt.

Der dritte Grund schließlich führt zurück zur Entstehungsgeschichte des Buches. Das Thema des Konfliktes trifft in der angelsächsischen Welt ohnehin auf ein unmittelbareres Echo als in Deutschland. Es wird auch nicht gleich Partei (,,Konfliktpädagogik"), sondern paßt in eine Tradition, in der etwa S. M. Lipset ganz unbefangen die Rede vom „demokratischen Klassenkampf" einführen konnte. Hier allerdings beginnt dann eine Fragekette, die das Klassenbuch von Anfang an begleitet hat.

Es gibt einen weiteren Kulturbereich, in dem Soziale Klassen und Klassenkonflikt eine andauernde Wirkung gehabt hat, das ist der italienisch-iberisch-lateinamerikanische. Da haben Übersetzun- gen bald zu Taschenbuchausgaben geführt, und Nachdrucke erscheinen bis auf den heutigen Tag; da gibt es auch eine nicht unbeträchtliche Sekundärliteratur. Man geht wohl nicht fehl in der Annahme, daß in diesen Ländern das Interesse an dem Theoriestück andere Gründe hat als in der angelsächsischen Welt. Hier wirkt die Tatsache, $\mathrm{da} B$ es sich sozusagen um einen Marxismus ohne Marx handelt. Es wird also die Klassenterminologie und damit die Zugehörigkeit zur Marxschen Denktradition beibehalten, zugleich aber eine Analyse entworfen, die ihre Vertreter nicht dem Anwurf des Marxismus aussetzt. Das hat etwas zu tun mit der Fragekette, die das Klassenbuch begleitet hat.

Denn man könnte ja fragen, warum der Begriff der Klasse hier überhaupt beibehalten wird. Handelt es sich nicht im Grunde um eine Theorie des Konflikts, die auch ohne „Klassen“ und „Klassenkonflikte" auskäme? Das ist nicht in dem Sinne gemeint, in dem Offe und andere den Klassenbegriff als ,selbst historisch“, also als an ein bestimmtes Stadium der kapitalistischen Entwicklung gekettet ansehen. Für den Erfahrungswissenschaftler beruht die Verwendung von Begriffen immer auf Entschlüssen; sie hat keine wie auch immer geartete innere Notwendigkeit. Nur liegt der methodische Kern des Versuches der Überwindung von Marx ja darin, eine allgemeinere Theorie $\mathrm{zu}$ finden, die sowohl die Ereignisse, die sich mit Marx verstehen lassen, als auch die, an denen Marx aufläuft, erklärt. Das ist eine Theorie des Konflikts, vielleicht eine des Herrschaftskonflikts, für die der Name Klassenkonflikt möglicherweise nicht nur unnötig, sondern wegen seiner historischen Assoziationen milde irreführend ist. Es reicht zu sagen, daß gesellschaftliche Veränderungen das Ergebnis von Auseinandersetzungen zwischen Gruppen sind, die ihre Kraft aus den obwaltenden sozialökonomischen Umständen beziehen (um eine oben verwendete Formulierung noch einmal aufzunehmen). Immer kämpft eine Gruppe im Namen zukünftiger Möglichkeiten gegen eine andere, die vorhandene Strukturen verteidigt.

In „Conflict After Class" habe ich die Verallgemeinerung der Theorie bis an ihre Grenze getrieben. Seinerzeit habe ich einen Begriff gesucht, der noch allgemeiner ist als „Konflikt" und die Kraft bezeichnet, die in Konflikten, aber auch in individuellem Handeln, etwa in Mobilität, ihren Ausdruck findet, eine Kraft der Unruhe sozusagen. Damals habe ich das englische Wort contest verwendet, bin im Deutschen mit Kants Wort „Antagonismus“ 
umgegangen. Danach gäbe es dann eine allgemeine soziale Kraft der Unruhe, die in individueller Konkurrenz wie in solidarischen Konflikten, im Sonderfall auch in Klassenkonflikten à la Marx ihren Ausdruck finden kann.

In einem späteren (unveröffentlichten) Manuskript, Eclipse of Modernity, habe ich eine andere Linie der Überlegung verfolgt. Im Klassenbuch schien mir der Konflikt, und zwar noch der latente Konflikt zwischen Kategorien mit gemeinsamer Interessenlage, zu sehr in der Luft zu schweben. $\mathrm{Zu}$ wenig Aufmerksamkeit war dem Marxschen Verhältnis von Produktivkräften und Produktionsverhältnissen gewidmet worden. Dabei ließe sich auch dieser Gedanke aus seiner Zeitbindung befreien und verallgemeinern. Wenn sich „soziale Kräfte" und "soziale Strukturen“ in einer Weise identifizieren ließen, die einen Bezug auf Konflikte hat, dann besäße man vielleicht ein analytisches Instrumentarium für das Verständnis sozialen Wandels, das noch ergiebiger ist als die Theorie des Konflikts.

Heute scheint mir ein Kernproblem moderner Gesellschaften darin zu liegen, daß in ihnen der Gesellschaftsvertrag selbst zum Thema wird. Die Ge- sellschaft erfüllter Staatsbürgerrechte - citizenship rights - hat begonnen, nicht unbeträchtliche Mengen, vielleicht auch Gruppen, hinauszudefinieren, auszugrenzen. Diese wehren sich (einstweilen? systematisch auf Grund ihrer Position?) eher individuell und situationell als durch solidarische soziale Konflikte. Sie leugnen für sich den Gesellschaftsvertrag und verstärken damit dessen Fragwürdigkeit; sie stellen Legitimitätsfragen. Daß hier neue Fronten der Unruhe, vielleicht auch des Konflikts entstehen, liegt auf der Hand. Gibt es eine Theorie, mit der sich sowohl der „Klassische Klassenkampf“ als auch der „demokratische Klassenkampf" als auch die individualisierte Unruhe als auch die neue Auseinandersetzung um den Gesellschaftsvertrag fassen läßt?

Ich weiß es nicht. Aber ich würde vermuten, daß es sich lohnt, in dieser Absicht jenen Ansatz weiterzuentwickeln, der in Class and Class Conflict seinen ersten Niederschlag gefunden hat. Ich gehe also vorerst davon aus, daß es sich keineswegs um ein ,relativ abgeschlossenes Theorie- und Analysestück", sondern im Gegenteil um einen fruchtbaren, offenen Ansatz handelt. Der Beweis ist allerdings erst noch anzutreten. 